This item was submitted to Loughborough's Research Repository by the author.

Items in Figshare are protected by copyright, with all rights reserved, unless otherwise indicated.

\title{
Low-temperature proximity effect in clean metals with repulsive electron-
} electron interaction

PLEASE CITE THE PUBLISHED VERSION

PUBLISHER

(C) American Physical Society

VERSION

VoR (Version of Record)

LICENCE

CC BY-NC-ND 4.0

\section{REPOSITORY RECORD}

Alexandrov, A.S., and V.V. Kabanov. 2019. "Low-temperature Proximity Effect in Clean Metals with Repulsive Electron-electron Interaction”. figshare. https://hdl.handle.net/2134/4277. 
This item was submitted to Loughborough's Institutional Repository (https://dspace.lboro.ac.uk/) by the author and is made available under the following Creative Commons Licence conditions.

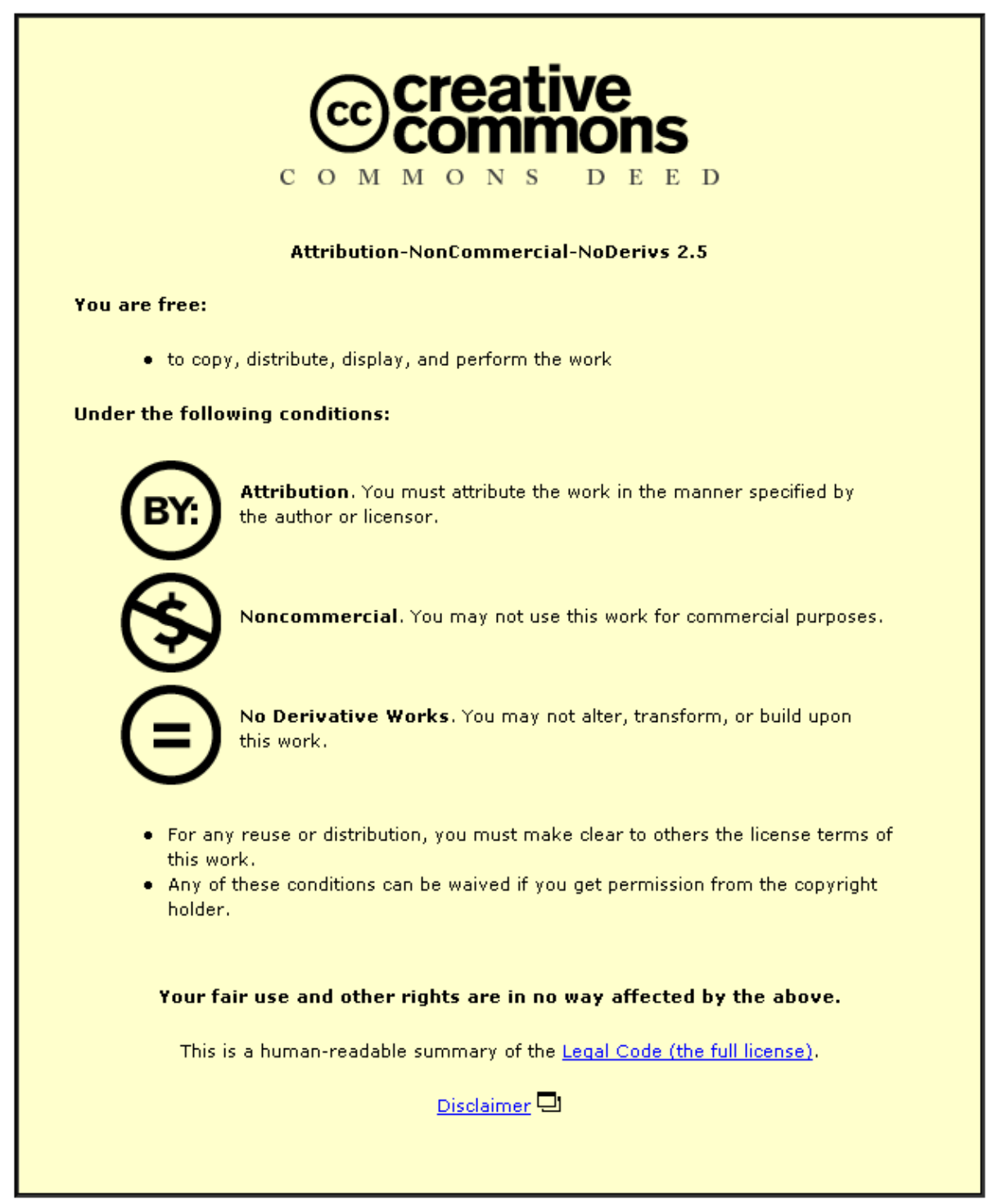

For the full text of this licence, please go to: http://creativecommons.org/licenses/by-nc-nd/2.5/ 


\title{
Low-temperature proximity effect in clean metals with repulsive electron-electron interaction
}

\author{
A. S. Alexandrov and V. V. Kabanov \\ Department of Physics, Loughborough University, Loughborough LE11 3TU, United Kingdom \\ and Josef Stefan Institute 1001, Ljubljana, Slovenia
}

(Received 7 October 2008; published 27 October 2008)

\begin{abstract}
Theories of proximity effect in layered superconductor-normal-metal (SN) structures usually deal with a hypothetic normal metal with no direct repulsive interaction between electrons and with finite temperatures often close to the superconductor critical temperature. We present an asymptotic solution of the Gor'kov equations in the opposite low-temperature limit for a clean normal metal with a repulsive interaction between electrons. The order parameter in the metal exhibits a power-law decay, $\Delta(x) \propto \xi / x$, as a function of the distance from the SN boundary, $x$, with a proximity length $\xi$ strongly depending on the repulsive interaction.
\end{abstract}

DOI: $10.1103 /$ PhysRevB.78.132510

PACS number(s): 71.38. $-\mathrm{k}, 72.15 . J f, 74.40 .+\mathrm{k}, 74.72 .-\mathrm{h}$

In recent years investigations of different superconductornormal-metal (SN) structures $^{1}$ have gone through a vigorous revival. In particular, superconductor-ferromagnet structures, ${ }^{2-6}$ cuprate junctions, ${ }^{7-9}$ and mesoscopic SN structures ${ }^{10-13}$ have been experimentally studied and addressed theoretically.

The superconducting order parameter penetrates into a bulk normal metal across the SN boundary. The microscopic theory of this proximity effect has been developed at finite temperatures (for reviews see Refs. 14 and 15), using the Eilenberger formalism ${ }^{16}$ and the semiclassical Usadel approximation ${ }^{17}$ for solving the Gor'kov equations ${ }^{18}$ close to and below $T_{c}$, in particular in the dirty limit. ${ }^{19,20}$ The clean case has been studied for a hypothetic normal metal with no interaction between electrons, ${ }^{21,22}$ and for a "normal" metal with a weak attractive interaction and reduced critical temperature. ${ }^{8,23,24}$ Reference 24 numerically calculated a decay of the order parameter also in a single particular case of a repulsive interaction at finite temperatures. An exponential decay of $\Delta(x) \propto \exp (-x / \xi)$, as a function of the distance $x$ from the SN boundary, has been found with the proximity length $\xi \propto 1 / T$ in the clean limit, and $\xi \propto 1 / \sqrt{T}$ in the dirty limit. ${ }^{1,14,15,20}$ Extensive theoretical investigations of the proximity effect have been performed using numerical methods to solve self-consistently the Bogoliubov-de Gennes equations in clean ferromagnet-superconductor structures with an exchange interaction. ${ }^{4}$ It was shown that for low temperatures the pair amplitude in the normal metal decays approximately as the inverse of the distance from the interface.

To the best of our knowledge a role of the direct repulsive interaction in the normal clean metal at low temperatures has not been addressed, while its effect on the proximity length and the Josephson current has been found only marginal in the dirty case. ${ }^{19}$ Here we present an asymptotic solution of the Gor'kov equations for the SN boundary between a bulk superconductor and a bulk clean normal metal with the repulsion between electrons at low temperatures.

It is convenient to Fourier transform the Matsubara normal, $\mathcal{G}_{\omega}\left(\mathbf{r}-\mathbf{r}^{\prime}, x, x^{\prime}\right)$, and anomalous, $\mathcal{F}_{\omega}^{+}\left(\mathbf{r}-\mathbf{r}^{\prime}, x, x^{\prime}\right)$, Green's functions (GFs) along the boundary, $\mathbf{r}=\{y, z\}$ with the wave vector $\mathbf{k}=\left\{k_{y}, k_{z}\right\}$,

$$
\mathcal{G}_{\omega}\left(\mathbf{r}-\mathbf{r}^{\prime}, x, x^{\prime}\right)=(2 \pi)^{-2} \int d \mathbf{k} \mathcal{G}_{\omega, k}\left(x, x^{\prime}\right) \exp \left[i \mathbf{k} \cdot\left(\mathbf{r}-\mathbf{r}^{\prime}\right)\right],
$$

$$
\mathcal{F}_{\omega}^{+}\left(\mathbf{r}-\mathbf{r}^{\prime}, x, x^{\prime}\right)=(2 \pi)^{-2} \int d \mathbf{k} \mathcal{F}_{\omega, k}^{+}\left(x, x^{\prime}\right) \exp \left[i \mathbf{k} \cdot\left(\mathbf{r}-\mathbf{r}^{\prime}\right)\right] .
$$

The Gor'kov equations are derived using equations of motion for the Matsubara operators as

$$
\begin{gathered}
\frac{1}{2 m}\left(a^{2}+\frac{\partial^{2}}{\partial x^{2}}\right) \mathcal{G}_{\omega, k}\left(x, x^{\prime}\right)+\Delta(x) \mathcal{F}_{\omega, k}^{+}\left(x, x^{\prime}\right)=\delta\left(x-x^{\prime}\right), \\
\frac{1}{2 m}\left(a^{* 2}+\frac{\partial^{2}}{\partial x^{2}}\right) \mathcal{F}_{\omega, k}^{+}\left(x, x^{\prime}\right)-\Delta^{*}(x) \mathcal{G}_{\omega, k}\left(x, x^{\prime}\right)=0,
\end{gathered}
$$

$$
\frac{1}{2 m}\left(a^{2}+\frac{\partial^{2}}{\partial x^{\prime 2}}\right) \mathcal{G}_{\omega, k}\left(x, x^{\prime}\right)+\Delta^{*}\left(x^{\prime}\right) \mathcal{F}_{-\omega, k}^{+*}\left(x, x^{\prime}\right)=\delta\left(x-x^{\prime}\right),
$$

$$
\frac{1}{2 m}\left(a^{* 2}+\frac{\partial^{2}}{\partial x^{\prime 2}}\right) \mathcal{F}_{-\omega, k}^{+*}\left(x, x^{\prime}\right)-\Delta\left(x^{\prime}\right) \mathcal{G}_{\omega, k}\left(x, x^{\prime}\right)=0 .
$$

Here $m, k_{F}$ are the electron effective mass and the Fermi momentum, respectively, which are taken the same in the superconductor and in the normal metal for mathematical transparency, $a^{2}=2 m\left(i \omega-\xi_{k}\right)$ with $\xi_{k}=\left(k^{2}-k_{F}^{2}\right) / 2 m$ and $a$ $=\operatorname{sign}(\omega) \sqrt{m\left(\rho+\xi_{k}\right)}+i \sqrt{m\left(\rho-\xi_{k}\right)}, \quad \rho \equiv+\sqrt{\omega^{2}+\xi_{k}^{2}}, \quad \omega=2 \pi T$ $(n+1 / 2)$ is the Matsubara frequency $(n=0, \pm 1, \pm 2, \ldots)$. We use $\hbar=k_{B}=1$ here and below, and $\mathcal{F}_{-\omega, k}^{+*}\left(x, x^{\prime}\right)$ $=\mathcal{F}_{\omega, k}\left(x, x^{\prime}\right)$. The superconducting order parameter

$$
\Delta(x) \equiv-V(x) T(2 \pi)^{-2} \sum_{\omega} \int d \mathbf{k} \mathcal{F}_{\omega, k}(x, x)
$$

is a solution of an integral equation,

$$
\Delta(x)=-V(x) \int_{-\infty}^{\infty} d x^{\prime} \mathcal{G}_{-\omega, k}^{(0)}\left(x, x^{\prime}\right) \Delta\left(x^{\prime}\right) \mathcal{G}_{\omega, k}\left(x, x^{\prime}\right),
$$

where $V(x)$ is the electron-electron contact interaction, which is negative (attractive) in the superconductor at $x<0$, and positive (repulsive) in the normal metal, $V(x) \equiv V_{c}>0$, at $x$ $>0$ [Fig. 1]. The GF of a bulk normal metal is 


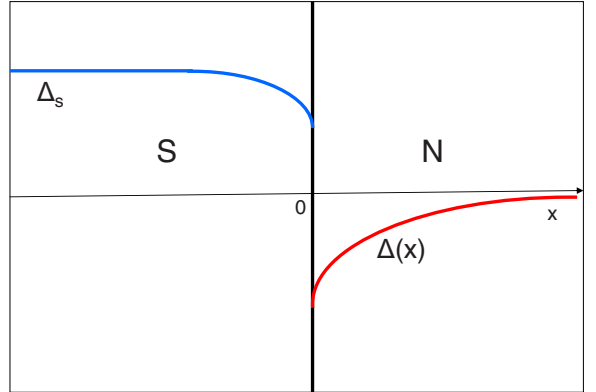

FIG. 1. (Color online) The order-parameter profile near the boundary between a superconductor $(\mathrm{S})$ and a normal metal $(\mathrm{N})$ with the repulsive electron-electron interaction.

$$
\mathcal{G}_{\omega, k}^{(0)}\left(x, x^{\prime}\right)=\frac{m}{i a} \exp \left(i a\left|x-x^{\prime}\right|\right)
$$

The Gor'kov equations are supplemented by the boundary conditions, $\Delta(-\infty) \equiv \Delta_{s}, \Delta(\infty)=0$, and all GFs should be continuous with respect to $x, x^{\prime}$ together with their first derivatives at the boundary $x=0$ or $x^{\prime}=0$.

To solve the integrodifferential system of Eqs. (1)-(6) let us assume that the repulsive interaction in the normal metal significantly reduces the order parameter, so that the latter is small, $\Delta(x) \ll \Delta_{s}$, far away from the boundary at $x \gg \xi$, where the proximity length $\xi$ is small compared with the superconductor coherence length, $\xi_{s}, \xi \ll \xi_{s}$. That allows us to use in Eq. (6) a solution, $\mathcal{G}_{\omega, k}\left(x^{\prime}, x\right) \approx \mathcal{G}_{\omega, k}^{s t}\left(x^{\prime}, x\right)$ of the Gor'kov Eqs. (1) -(4) with a steplike order parameter $\Delta(x)=\Delta_{S} \Theta(-x)$, where $\Theta(x)$ is the Heaviside step function. This solution can be readily obtained by matching GFs and their derivatives at the boundaries between four domains: a superconductor domain $S$, where both arguments are negative $x, x^{\prime}<0$, two mixed domains $M$ with $x<0<x^{\prime}$ or $x^{\prime}<0<x$, and a normal domain $N$, where $x, x^{\prime}>0$ [Fig. 2].

In the normal domain the solution is found as (see also Refs. 21 and 23)

$$
\mathcal{G}_{\omega, k}^{s t N}\left(x, x^{\prime}\right)=\mathcal{G}_{\omega, k}^{(0)}\left(x, x^{\prime}\right)+A e^{i a\left(x+x^{\prime}\right)},
$$

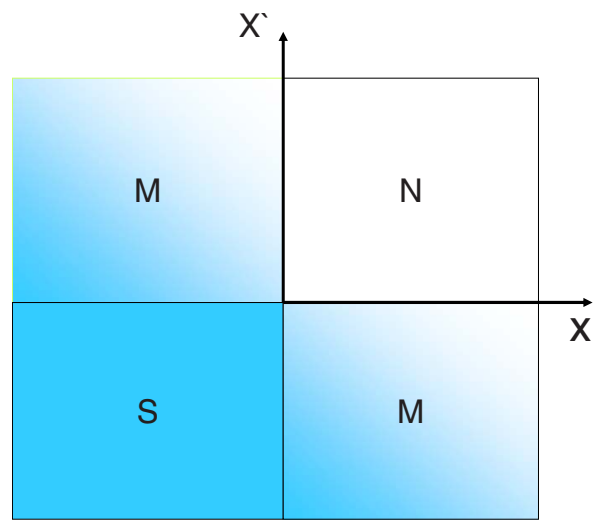

FIG. 2. (Color online) Four domains with different solutions for GFs with the steplike order parameter $\Delta(x)=\Delta_{s} \Theta(-x)$.

$$
\mathcal{F}_{\omega, k}^{+s t N}\left(x, x^{\prime}\right)=B e^{i\left(a x^{\prime}-a^{*} x\right)},
$$

and in one of the mixed domains, $x^{\prime}<0<x$, as

$$
\begin{gathered}
\mathcal{G}_{\omega, k}^{s t M}\left(x, x^{\prime}\right)=C \frac{2 m \Delta_{s}}{\left(b^{2}-a^{2}\right)} e^{i\left(a x-b x^{\prime}\right)}+D \frac{2 m \Delta_{s}}{\left(b^{* 2}-a^{2}\right)} e^{i\left(a x+b^{*} x^{\prime}\right)}, \\
\mathcal{F}_{\omega, k}^{+s t M}\left(x, x^{\prime}\right)=C^{*} e^{i\left(b^{*} x^{\prime}-a^{*} x\right)}+D^{*} e^{-i\left(b x^{\prime}+a^{*} x\right)}
\end{gathered}
$$

where $A, B, C$, and $D$ are constants and $b^{2}=2 m\left(i \epsilon-\xi_{k}\right), \Im b$ $>0, \epsilon=\sqrt{\omega^{2}+\Delta_{s}^{2}}$. The constants are found from

$$
\begin{aligned}
\mathcal{G}_{\omega, k}^{s t N}(x, 0) & =\mathcal{G}_{\omega, k}^{s t M}(x, 0), \\
\mathcal{F}_{\omega, k}^{+s t N}(x, 0) & =\mathcal{F}_{\omega, k}^{+s t M}(x, 0)
\end{aligned}
$$

and from

$$
\begin{gathered}
\frac{\partial}{\partial x^{\prime}} \mathcal{G}_{\omega, k}^{s t N}\left(x, x^{\prime}\right)=\frac{\partial}{\partial x^{\prime}} \mathcal{G}_{\omega, k}^{s t M}\left(x, x^{\prime}\right), \\
\frac{\partial}{\partial x^{\prime}} \mathcal{F}_{\omega, k}^{+s t N}\left(x, x^{\prime}\right)=\frac{\partial}{\partial x^{\prime}} \mathcal{F}_{\omega, k}^{+s t M}\left(x, x^{\prime}\right)
\end{gathered}
$$

at $x^{\prime}=0$.

In particular we find

$$
A=\frac{m}{i a}\left\{\frac{2 a\left[\left(a^{*}+b^{*}\right)(\epsilon+\omega)+\left(a^{*}-b\right)(\epsilon-\omega)\right]}{|a+b|^{2}(\epsilon+\omega)+\left|a-b^{*}\right|^{2}(\epsilon-\omega)}-1\right\},
$$

and

$$
B=2 m \Delta_{s} \frac{b+b^{*}}{|a+b|^{2}(\epsilon+\omega)+\left|a-b^{*}\right|^{2}(\epsilon-\omega)} .
$$

Integrating over the normal region, $x^{\prime}>0$ in Eq. (6) one can keep only the first "normal" term of Eq. (8) at sufficiently large $x>\xi$, while integrating over the superconductor region, $x^{\prime}<0$, one can use directly Eq. (9) and the definition of $\Delta(x)$, Eq. (5) to obtain

$$
\begin{aligned}
\widetilde{\Delta}(x)= & -V_{c} \operatorname{Tm} \sum_{\omega} \int \frac{d \mathbf{k}}{(2 \pi)^{2}} \\
& \times\left[u e^{-2 x \Im a}+\frac{m}{|a|^{2}} \int_{0}^{\infty} d x^{\prime} \tilde{\Delta}\left(x^{\prime}\right) e^{-2\left|x-x^{\prime}\right| \Im a}\right],
\end{aligned}
$$

where $\widetilde{\Delta}=\Delta(x) / \Delta_{s}$ is the reduced order parameter in the normal metal, and $u=B / m \Delta_{s}$.

At finite temperatures and $x \gg v_{F} /(2 \pi T)$ the main contribution to the Matsubara sum in Eq. (14) comes from the $n$ $=0$ term, so that the order parameter has the conventional exponential asymptotic, $\Delta(x) \propto \exp \left(-2 \pi T x / v_{F}\right)$, where $v_{F}$ $=k_{F} / m$ is the Fermi velocity. At sufficiently low and zero temperatures one has $x \ll v_{F} /(2 \pi T)$ for any size of the normal region, so that the exponential asymptotic is replaced by some power decay. ${ }^{25}$ To find the power we replace the Matsubara sum by an integral over $\omega$, and integrating over momentum and frequency in Eq. (14), we obtain an integral equation for the reduced order parameter, $\widetilde{\Delta}(x)=\Delta(x) / \Delta_{s}$, 


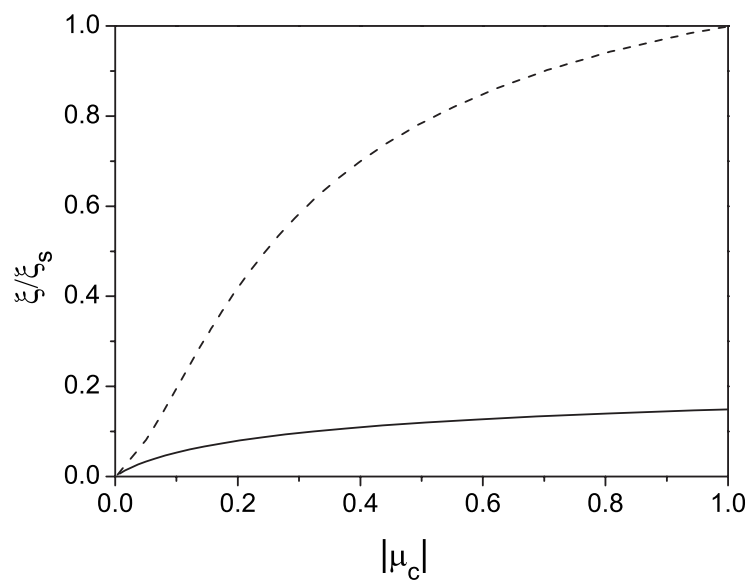

FIG. 3. SN proximity length $\xi$ (in units of the superconductor coherence length) as a function of the repulsive pseudopotential $\mu_{c}$ (solid line) and of the attractive potential (dashed line).

$$
\frac{\xi_{s}}{x}+\int_{0}^{\infty} d x^{\prime} \frac{\tilde{\Delta}\left(x^{\prime}\right)}{\left|x^{\prime}-x\right|}=-\frac{\tilde{\Delta}(x)}{\mu_{c}},
$$

where $\mu_{c}=V_{c} m k_{F} / 4 \pi^{2}$ is the repulsion pseudopotential, and $\xi_{s}=v_{F} / 2 \Delta_{s}$. One can satisfy Eq. (15) with a solution decaying as inverse distance from the boundary,

$$
\widetilde{\Delta}(x)=-\xi / x .
$$

Substituting Eq. (16) into Eq. (15) yields

$$
\tilde{\xi}=\frac{\mu_{c}}{1+\mu_{c} \int_{0}^{\infty} d t|t| t-1 \mid},
$$

where $\tilde{\xi}=\xi / \xi_{s}$ is the dimensionless proximity length.

The logarithmic divergency of the integral in Eq. (17) is an artifact of the step-function approximation [Eqs. (8) and (9)]. We cut the divergency by excluding small regions, $t$ $<t_{\text {min }} \ll 1$ and $|t-1|<t_{\text {min }}$ from the integral, where the stepfunction approximation fails because $\widetilde{\Delta}(x)$ becomes relatively large. We chose $t_{\min }=\tilde{\xi}$ since $\tilde{\xi}$ is the only dimensionless parameter in the normal region which corresponds to the cutoff of the integral over $x^{\prime}$ in Eq. (15) at a cutoff length $x^{\prime}>l_{\min }=\widetilde{\xi} x$, proportional to $x$. The result is a transcendental equation for $\tilde{\xi}$,

$$
\tilde{\xi}=\frac{\mu_{c}}{1-3 \mu_{c} \ln (\tilde{\xi})} .
$$

The proximity length is shown in Fig. 3 as a function of the repulsion $\mu_{c}$. It strongly depends on the repulsion with a maximum value $\widetilde{\xi}_{\max } \approx 0.22$ at $\mu_{c}=\infty .{ }^{26}$ The magnitude of $\widetilde{\xi}$ is small at any $\mu_{c}$, which justifies our step-function approximation [Eqs. (8) and (9)] for solving the problem.

To verify the self-consistency of the approximation one can estimate the correction, $\delta \mathcal{G}_{\omega, k}\left(x, x^{\prime}\right)$, to GF [Eq. (8)] due to the finite order parameter in the normal region; at large $x, x^{\prime}>0$

$$
\delta \mathcal{G}_{\omega, k}\left(x, x^{\prime}\right)=-\int_{0}^{\infty} d x^{\prime \prime} \mathcal{G}_{\omega, k}^{(0)}\left(x, x^{\prime \prime}\right) \Delta\left(x^{\prime \prime}\right) \mathcal{F}_{\omega, k}^{+s t N}\left(x^{\prime \prime}, x^{\prime}\right)
$$

Using Eqs. (9) and (16) one obtains $\delta \mathcal{G}_{\omega, k}\left(x, x^{\prime}\right)$ $\propto \widetilde{\xi} \ln \left(x / l_{\min }\right)$, which is small as $\widetilde{\xi} \ln (1 / \widetilde{\xi}) \ll 1$ at any $x$ with our choice of the cutoff length, $l_{\min }=\widetilde{\xi} x$. Another possible choice of the cutoff length $l_{\min }=\xi$ does not change the order parameter in a wide region $\xi_{s}<x<\xi_{s} / \widetilde{\xi}$ because the singularity is logarithmically weak. We note that the order parameter becomes so small at very large distance from the boundary, that a mean-field approximation used in Eqs. (1)-(4) may break down. ${ }^{27}$

While the order parameter saturates at a small value for any fixed $x$ in the normal region, the pair wave function, $\mathcal{F}_{\omega, k}^{N}\left(x, x^{\prime}\right)$, decreases as $1 / \mu_{c}$ at large repulsion. At first glance the existence of finite pair correlations in the repulsive normal metal looks surprising because it leads to a finite increase in the repulsive potential energy of the whole SN system. In particular, an individual real-space pair of electrons bound by some attractive potential on one side of the boundary would stay at an infinite distance from the boundary with the repulsive interaction (zero proximity effect). However, the electron-density homogeneity in metals creates a quantum pressure on pairs pushing them across the boundary. The reason for the failure of the pair wave function to die off is that there is simply no pair-breaking mechanism for disrupting any correlation that drift across the boundary as in the case of a hypothetical normal metal with no interaction. ${ }^{21}$ With the power-law order parameter, $\Delta(x) \propto 1 / x$, an increase in the potential energy proportional to $\Delta^{2}(x)$ is finite when it is integrated over the whole normal region. Compared with the steplike order parameter this increase is compensated by a lowering of the kinetic energy near the boundary.

It is instrumental to compare the $\mathrm{SN}$ proximity effect in the repulsive normal metal with the effect in a normal metal with a small attractive potential between electrons at low but finite temperatures above the transition temperature, $T_{c n}$ $\left(\ll T_{c}\right)$, of the normal metal. Solving Eq. (15) with a negative $\mu_{c}$ yields a positive order parameter $\widetilde{\Delta}_{\text {att }}(x)=\xi_{\text {att }} / x$ with the proximity length,

$$
\frac{\xi_{\mathrm{att}}}{\xi_{s}} \equiv \widetilde{\xi}_{\mathrm{att}}=\frac{\left|\mu_{c}\right|}{1+3\left|\mu_{c}\right| \ln \left(\widetilde{\xi}_{\mathrm{att}}\right)},
$$

shown in Fig. 3 as the dashed line. With increasing attraction the proximity length increases linearly as in the case of the repulsion, but much faster saturating at $\widetilde{\xi}_{\text {att }} \approx 1.2$ for the values of the interaction which are well beyond the stepfunction (and BCS) approximations. The power law decay of the order parameter holds for the region $x \ll v_{F} /(2 \pi T)$, but different from the repulsive case only for finite temperatures, which are much higher than $T_{c n}$ and smaller than $T_{c}$.

It is also worthwhile to mention that the normal Fermi liquid with the hard-core repulsion between fermions becomes a $p$-wave ${ }^{28}$ or a $d$-wave ${ }^{29}$ superfluid at very low temperatures (on the millidegrees Kelvin scale) due to the Frie- 
del oscillations ${ }^{30}$ of the particle-particle interaction potential caused by screening. This unconventional pairing should not affect our results for the proximity effect with the conventional $s$-wave superconductor as long as there is no symmetry-breaking mechanism. On the other hand if such a mechanism is involved (e.g., the spin-orbit coupling), the inhomogeneous $s$-wave order parameter can generate a secondary order parameter of another symmetry. ${ }^{31-33}$ Finally, the order parameter in a dirty normal metal should also exhibit some power-law decay at temperatures lower than the Thouless energy, but the power could be different from $1 / x$ of the clean metal. ${ }^{20,34}$

In summary we have solved a long-standing problem of the low-temperature proximity effect in a clean normal metal with repulsive interaction between electrons. We have found the power-law decay of the order parameter with the characteristic proximity length significantly reduced with respect to the superconductor coherence length by the repulsive interaction in the normal metal. Our predictions could be tested on the SN structures with a paramagnetic clean normal metal close to the ferromagnetic instability where the Coulomb repulsion is strong enough.

We thank A. F. Andreev, A. A. Golubov, L. P. Gor'kov, D. Khmelnitskii, M. Yu. Kuprianov, K. K. Likharev, L. A. Maksimov, and A. V. Paraskevov for illuminating discussions and constructive suggestions. The work was supported by EPSRC (U.K.) (Grant No. EP/D035589/1) and by the Slovenian Research Agency (ARRS) (Grant No. 430-66/200717).
${ }^{1}$ P. G. de Gennes, Rev. Mod. Phys. 36, 225 (1964).

${ }^{2}$ A. I. Buzdin, Rev. Mod. Phys. 77, 935 (2005), and references therein.

${ }^{3}$ F. S. Bergeret, A. F. Volkov, and K. B. Efetov, Phys. Rev. B 64, 134506 (2001); 65, 134505 (2002).

${ }^{4}$ K. Halterman and O. T. Valls, Phys. Rev. B 66, 224516 (2002).

${ }^{5}$ V. Zdravkov, A. Sidorenko, G. Obermeier, S. Gsell, M. Schreck, C. Muller, S. Horn, R. Tidecks, and L. R. Tagirov, Phys. Rev. Lett. 97, 057004 (2006).

${ }^{6}$ A. S. Vasenko, A. A. Golubov, M. Yu. Kupriyanov, and M. Weides, Phys. Rev. B 77, 134507 (2008).

${ }^{7}$ I. Bozovic, G. Logvenov, M. A. J. Verhoeven, P. Caputo, E. Goldobin, and M. R. Beasley, Phys. Rev. Lett. 93, 157002 (2004), and references therein.

${ }^{8}$ J. Quintanilla, L. N. Oliveira, and K. Capelle, Phys. Rev. Lett. 90, 089703 (2003).

${ }^{9}$ A. S. Alexandrov, Phys. Rev. B 75, 132501 (2007).

${ }^{10}$ C. J. Lambert and R. Raimondi, J. Phys.: Condens. Matter 10, 901 (1998).

${ }^{11}$ W. Belzig, F. K. Wilhelm, C. Bruder, G. Schön, and A. D. Zaikin, Superlattices Microstruct. 25, 1251 (1999).

${ }^{12}$ F. B. Muller-Allinger and A. C. Mota, Phys. Rev. Lett. 84, 3161 (2000).

${ }^{13}$ A. V. Galaktionov and A. D. Zaikin, Phys. Rev. B 67, 184518 (2003).

${ }^{14}$ G. Deutscher and P. G. de Gennes, in Superconductivity, edited by R. D. Parks (Marcel Dekker, New York, 1969), Vol. 2, p. 1005.

${ }^{15}$ K. K. Likharev, Rev. Mod. Phys. 51, 101 (1979).
${ }^{16}$ G. Eilenberger, Z. Phys. 214, 195 (1968).

${ }^{17}$ K. D. Usadel, Phys. Rev. Lett. 25, 507 (1970).

${ }^{18}$ L. P. Gor'kov, Zh. Eksp. Teor. Fiz. 34, 735 (1958) [Sov. Phys. JETP 7, 505 (1958)]; Zh. Eksp. Teor. Fiz. 36, 1918 (1959) [Sov. Phys. JETP 9, 1364 (1958)].

${ }^{19}$ M. Yu. Kuprianov, K. K. Likharev, and V. F. Lukichev, Physica B \& C 108, 1001 (1981); Zh. Eksp. Teor. Fiz. 83, 431 (1982) [Sov. Phys. JETP 56, 235 (1982)].

${ }^{20}$ P. Dubos, H. Courtois, B. Pannetier, F. K. Wilhelm, A. D. Zaikin, and G. Schön, Phys. Rev. B 63, 064502 (2001).

${ }^{21}$ D. S. Falk, Phys. Rev. 132, 1576 (1963).

${ }^{22}$ A. D. Zaikin and G. F. Zharkov, Phys. Lett. A 95, 331 (1983).

${ }^{23}$ L. A. Maksimov and A. P. Potapenko (unpublished).

${ }^{24}$ L. Covaci and F. Marsiglio, Phys. Rev. B 73, 014503 (2006).

${ }^{25}$ K. K. Likharev (private communication).

${ }^{26}$ In fact, the value of $\mu_{c}$ is restricted from above, $\mu_{c}<1$, because of the Stoner ferromagnetic instability of the normal metal at $\mu_{c}>1$.

${ }^{27}$ L. P. Gor'kov (private communication).

${ }^{28}$ W. Kohn and J. M. Luttinger, Phys. Rev. Lett. 15, 524 (1965).

${ }^{29}$ A. S. Alexandrov and A. A. Golubov, Phys. Rev. B 45, 4769 (1992).

${ }^{30}$ H. Friedel, Adv. Phys. 3, 446 (1954).

${ }^{31}$ V. V. Kabanov, Phys. Rev. B 76, 172501 (2007).

${ }^{32}$ L. P. Gor'kov and E. I. Rashba, Phys. Rev. Lett. 87, 037004 (2001).

${ }^{33}$ V. M. Edelstein, Phys. Rev. Lett. 75, 2004 (1995).

${ }^{34}$ M. Yu. Kuprianov and K. K. Likharev (unpublished). 